\title{
LA ACTIVIDAD FÍSICA DE LA COMUNIDAD RURAL: LA YMCA EN MÉXICO DURANTE LA DÉCADA DE LOS AÑOS 20
}

\author{
Lucía Martínez Moctezuma \\ Universidad Autónoma del Estado de Morelos - México \\ luciamm@uaem.mx
}

\begin{abstract}
RESUMEN
Después del movimiento revolucionario de 1910 en México, la Secretaría de Educación Pública puso en práctica una serie de proyectos educativos orientados a las necesidades de la población rural. Las Misiones Culturales lograron conjuntar a toda la comunidad en torno a una serie de actividades que, a través de la escuela, apuntaron el desarrollo físico y cultural de niños, mujeres y hombres, con la realización de festivales y encuentros deportivos en los que se conjugó el baile y el canto con la práctica del futbol, el basquetbol, el volibol y el beisbol. El objetivo de este trabajo es dar cuenta de estas prácticas novedosas que muestran la implementación de un modelo norteamericano de desarrollo físico en la escuela mexicana, el de la Young Men's Christian Association YMCA, en el que se implementan nuevas estrategias para integrar a la comunidad rural a partir de propuestas festivas y deportivas que apuntaban también a un disciplinamiento y administración del tiempo libre.
\end{abstract}

Palabras clave: YMCA. Actividad física. Profesor. Comunidad rural. Fotografía.

\section{THE PHYSICAL ACTIVITY OF THE RURAL COMMUNITY: THE YMCA IN MEXICO DURING THE DECADE OF THE 20 YEARS}

\begin{abstract}
After the revolutionary movement of 1910 in Mexico, the Ministry of Public Education put into practice a series of educational projects oriented to the needs of the rural population. The Cultural Missions managed to bring together the whole community around a series of activities that, through the school, aimed at the physical and cultural development of children, women and men, with the holding of festivals and sporting events in which they combined dancing and singing with the practice of soccer, basketball, volleyball and baseball. The objective of this work is to account for these novel practices that show the implementation of an American model of physical development in the Mexican school, the Young Men's Christian Association YMCA, in which new strategies are implemented to integrate the rural community based on festive and sports proposals that also pointed to a discipline and administration of free time.
\end{abstract}

Keywords: YMCA. Physical activity. Teacher and rural community. Photography.

\section{A ATIVIDADE FÍSICA DA COMUNIDADE RURAL: A ACM NO MÉXICO DURANTE A DÉCADA DOS 20 ANOS}

\section{RESUMO}

Após o movimento revolucionário de 1910 no México, o Ministério da Educação Pública pôs em prática uma série de projetos educacionais orientados para as necessidades da população rural. As Missões Culturais conseguiram reunir toda a comunidade em torno de uma série de atividades que, através da escola, visavam o desenvolvimento físico e cultural de crianças, 
mulheres e homens, com a realização de festivais e eventos esportivos nos quais se combinavam. dançando e cantando com a prática de futebol, basquete, vôlei e beisebol. $\mathrm{O}$ objetivo deste trabalho é dar conta dessas novas práticas que mostram a implementação de um modelo americano de desenvolvimento físico na escola mexicana, a Associação Cristã de Moços da YMCA, na qual novas estratégias são implementadas para integrar comunidade rural baseada em propostas festivas e esportivas que também apontavam para uma disciplina e administração do tempo livre.

Palavras-chave: YMCA. Atividade física. Professor e comunidade rural. Fotografia.

\section{L'ACTIVITÉ PHYSIQUE DE LA COMMUNAUTÉ RURALE: LE YMCA AU MEXIQUE PENDANT LA DÉCENNIE DES 20 ANS}

\section{RÉSUMÉ}

Après le mouvement révolutionnaire de 1910 au Mexique, le Ministère de l'Éducation Publique a mis en œuvre une série de projets éducatifs orientés vers les besoins de la population rurale. Les missions culturelles ont réussi à rassembler toute la communauté autour d'une série d'activités qui, à travers l'école, visaient le développement physique et culturel des enfants, des femmes et des hommes, avec la tenue de festivals et d'événements sportifs dans lesquels ils combinaient la danse et le chant avec la pratique du soccer, du basketball, du volleyball et du baseball. L'objectif de ce travail est de rendre compte de ces nouvelles pratiques qui montrent la mise en œuvre d'un modèle américain de développement physique à l'école mexicaine, l'Association des jeunes hommes chrétiens, YMCA, dans laquelle de nouvelles stratégies sont mises en œuvre pour intégrer le communauté rurale basée sur des propositions festives et sportives qui ont également visé la discipline et l'administration du temps libre.

Mots-clés: YMCA. Activité physique. Professeur et communauté rurale. Photographie

\section{INTRODUCCIÓN}

El tema de la historia de la educación física no ha merecido la atención de los investigadores (SPIVAK, 1981, p. 1), y menos desde la perspectiva del patrimonio histórico educativo, por esto resulta una excepción la reflexión planteada durante las VII Jornadas Científicas de la SEPHE y V Simposio Iberoamericano: Historia, Educación, Patrimonio Educativo (Universidad del País Vasco, San Sebastián, 29 de junio -1 de julio de 2016), en el que se conjugaron dos campos disciplinarios que se complementan: la Historia de la Educación y los estudios sobre el Patrimonio Histórico-Educativo. En este marco se presentaron una serie de trabajos que muestran la riqueza de este vínculo, ofreciendo otras lecturas y descubriendo diversas fuentes documentales que nos acercan a otra forma de mirar el Patrimonio Histórico Educativo:

[...] recuperar para el quehacer....un elemento clave, el espacio, entendiéndolo de un manera caleidoscópica... una diversidad de espacios por los que transcurre la vida de los alumnos y las alumnas...y adultos que soñaron alguna vez con un espacio ideal o de quienes recuerdan nostálgicamente los espacios por los que transcurrieron sus vidas 
en el pasado...se trata de una relación de los sujetos históricos con los espacios que les rodean y con los que establece una relación social, vital, escolar o imaginada y que producen relatos sobre experiencias de sus propias vidas, dejando un rastro patrimonial. (DÁVILA; NAYA, 2016, p. 19ss).

En esta perspectiva me interesa subrayar la relación entre el espacio físico (la adaptación de las instalaciones deportivas dentro de la comunidad rural) y el proyecto educativo posrevolucionario (las Misiones Culturales). Para poder llevarlo a cabo recurrí a los documentos originales que desde hace unos años alberga el Instituto de Ciencias de la Educación de la Universidad Autónoma de Morelos: manuales y cuadernos escolares, avances programáticos, informes de inspectores, cartas de profesores, de alumnos, gráficas, mapas y fotografías en los que se observa imágenes y representaciones del espacio escolar. Con la reproducción de diversos documentos originales localizados en el Fondo de la Secretaría de Educación Pública ${ }^{1}$, sobre todo de informes y oficios de inspectores y maestros, hemos identificado aquellos espacios relatados que nos acercan al cómo se desarrollaba la actividad educativa en otros tiempos. Para este trabajo he seleccionado algunos expedientes que dan cuenta de la actividad física en el Estado de Morelos, una zona rural de México. Las fotografías seleccionadas que acompañan este análisis fueron tomadas por los profesores que viajaban en los equipos de trabajo y que convivían con la comunidad rural durante 21 días. Después de una intensa labor académica, cultural y social, clausuraban sus actividades con torneos deportivos en los que niños y adultos mostraban los aprendizajes con encuentros atléticos, partidos de basquetbol y obras de teatro.

Se trata aún de pequeñas piezas del enorme rompecabezas que constituye la historia de la educación de un lugar que esperamos pronto sirva de base a un museo de la educación en el Estado de Morelos, un espacio académico al servicio de la investigación, la docencia y la difusión de nuestro pasado histórico-educativo.

\section{EL CONTEXTO. UN NUEVO PROYECTO EDUCATIVO PARA LA COMUNIDAD RURAL}

Cuando Moisés Sáenz ocupó el puesto de subsecretario en la Secretaría de Educación Pública (SEP), en 1925, se integró al equipo que impulsó el desarrollo de la Escuela Rural Mexicana. Junto a los profesores José Vasconcelos y Rafael Ramírez, Sáenz instrumentó el

\footnotetext{
${ }^{1}$ Gracias al apoyo de un proyecto de investigación nacional financiado por CONACyT, se elaboró la Guía del Archivo Histórico de la Secretaría de Educación Pública, Sección. Dirección de Educación Primaria en los Estados y Territorios. Serie. Escuelas Rurales Federales, Estado de Morelos, 1921-1979, que representa el primer instrumento de consulta para explorar los documentos de la entidad.
}

Rev. Iberoam. Patrim. Histórico-Educativo, Campinas (SP), v. 4, n. 1, p. 52-61, jan./jun. 2018 
proyecto de las Misiones Culturales en el que encontró una base para atender la integración social y cultural de los grupos indígenas a la nación mexicana, por lo que puso en práctica los conocimientos que había adquirido en sus viajes por Estados Unidos y Francia, donde conoció a personajes como el filósofo John Dewey y el misionero, lingüista especialista en lenguas nativas de Latinoamérica, William Cameron Townsend. Contar con "todo género de facilidades y libertad" le ofreció a Moisés Sáenz contar con una experiencia que le llevó a proponer un proyecto integral para el medio rural: partir de la escuela hacia la comunidad rural donde el maestro pudiera convertirse en un líder, como lo proponía la Young Men's Christian Association (YMCA) ${ }^{2}$.

Si bien este proyecto tuvo al inicio un carácter religioso se transformó en civilizatorio y en países como México, Brasil y Uruguay, encontró eco gracias a la diversidad de sus propuestas sociales y deportivas en las que se veía el medio ideal para preparar al hombre en una nueva época en la que se promovía por igual el descanso y el trabajo (MARTINEZ MOCTEZUMA, 2016). El modelo norteamericano se había inspirado del que se puso en práctica durante la Primera Guerra Mundial cuando Francia e Inglaterra necesitaron de mano de obra en razón de la movilización y las víctimas de guerra, que llevaron al contrato de cerca de 140 mil trabajadores chinos para suplir las vacantes en fábricas y puertos. En la integración de estos grupos asiáticos al medio europeo jugó un papel importante la YMCA, una asociación cristiana, creada en el Reino Unido en 1844 para favorecer el desarrollo espiritual, intelectual, físico y social de los habitantes. La YMCA organizó en los campamentos una serie de actividades deportivas y de entretenimiento entre las que se encontraban las proyecciones de cine, conciertos, funciones de teatro, fiestas y actividades educativas de gran utilidad porque el $80 \%$ de los trabajadores no sabía leer ni escribir ni hablar el idioma. Se estimuló la asistencia a la iglesia los días domingo y la lectura de la biblia en reuniones en las que se ponía en práctica una educación impregnada de moral cristiana. Los manuales escolares y las revistas fueron un importante medio de difusión para condenar malos hábitos como el consumo de tabaco, los juegos de azar y la prostitución. Para contrarrestar su efecto se estimularon las competencias deportivas y el basquetbol ${ }^{3}$. Se trataba de un esquema que privilegiaba la práctica del deporte

\footnotetext{
${ }^{2}$ En su carácter de Inspector General del Departamento Escolar y Asesor Técnico del Departamento Escolar, Moisés Sáenz fue comisionado reiteradamente para viajar a los Estados Unidos y cumplir con diversas actividades. Por ejemplo, entre el 27 de junio y el 7 de julio de 1923, dio una serie de conferencias a los estudiantes latinoamericanos y asistió al Congreso de Educación celebrado en San Francisco (AHSEP. Personal Sobresaliente. Expediente de Moisés Sáenz).

${ }^{3}$ Desde 1854 se fundó el International Committee of YMCA in USA and Canada con presencia en diferentes países. En 1885, China inauguró establecimientos escolares -liceos y universidades- que estuvieron bajo la dirección de profesorados norteamericanos o de chinos con estudios en Estados Unidos. Entre 1895 y 1920, la YMCA aumentó el número de sus establecimientos escolares: 2 en 1901 y 30 en 1920, con 32 mil adherentes. El YMCA y sus 
como antídoto para contrarrestar los efectos de la vida urbana pero además servía como vehículo para transmitir una serie de valores asociados a la identidad estadounidense en un país que había recibido cerca de 25 millones de inmigrantes entre 1865 y 1915.

Este modelo se incorporó a los programas escolares de educación primaria y secundaria en México, para instruir a los alumnos en la práctica de deportes como el beisbol y el basquetbol, un espacio donde se ejercitaron valores de la vida democrática y se transmitieron valores como la obediencia a las reglas, la cooperación, el reconocimiento a la excelencia (TORRES, 2011, p. 266). De manera general, este fue el programa de la YMCA que se implementó en las escuelas rurales en México y puede resumirse en tres aspectos: la formación de líderes sociales, la práctica del juego de conjunto y la habilidad para trabajar en cooperación con la comunidad.

El proyecto de las Misiones Culturales en México reunió a 4817 maestros rurales, 117 inspectores y un Director Federal por cada una de las treinta y un entidades del país. Cada misión formada por un equipo de profesores-misioneros se encargó de viajar a diferentes lugares del país para realizar tres tareas primordiales: detectar los problemas sociales de la comunidad, actualizar la formación de los maestros rurales que se ocupaban de la escuela primaria y dar un conocimiento básico a los habitantes de los pueblos con saberes agrícolas para los hombres y de economía doméstica e higiene, para las mujeres. En la práctica se articularon dos tipos de Misiones Culturales: ambulantes y permanentes. Las primeras, viajaban para actualizar la formación del maestro organizando Institutos de Perfeccionamiento durante aproximadamente cuatro semanas y estaban integrados por un profesor de Educación y Técnica de la Enseñanza, una trabajadora social, un agrónomo, una maestro de pequeñas industrias y un profesor de educación física. En las misiones permanentes su programa de acción era integral y abarcaba los aspectos social, económico y cultural para reforzar la formación de los niños. El equipo estaba formado por un doctor y una enfermera ayudante, una trabajadora social, un agrónomo, una matrona, un experto en industrias regionales, un carpintero y un albañil.

Una parte importante de las actividades de la Misión Cultural apuntaba al desarrollo físico y social de los habitantes de la comunidad rural, por esta razón, Moisés Sáenz se valdrá de la estructura de la YMCA, de la que será representante en México en estos años, para definir los programas de actividades. El interés de Sáenz por el deporte se remontaba a sus primeros

misioneros en China establecieron redes que fueron usadas para recrutar a los trabajadores chinos porque el War Office británico reconoció que eran los mejores propagadores de su filosofía pues contaban el respeto, afecto y confianza de la población quien no los veía como corruptos (MA, 2009, p. 103-107). 
años como director de la Escuela Nacional Preparatoria donde fomentó las actividades deportivas con la organización de los primeros Encuentros Atléticos Interescolares en 1919.

Sáenz consideraba que la escuela debía ofrecer al estudiante una serie de actividades físicas ${ }^{4}$ porque el campo de juegos era tan importante como el aula:

[...] y era en el vaivén de los deportes, en el jadeo del esfuerzo, en el apremio de la competencia, en la congoja real de la ficticia lucha, (que se) encuentra expresión filogenética toda la raza humana...el campo de juegos está salvando a la escuela moderna de la bancarrota de la ciencia libresca [...] (SÁENZ, 1920, p. 14).

\section{LAS MISIONES CULTURALES, UN PROYECTO EDUCATIVO E INTEGRAL}

El propósito original de las Misiones Culturales fue el mejoramiento profesional de los maestros federales y, consecuentemente la mejora cultural e higiénica de las comunidades rurales. Para actualizar a los profesores se crearon los Institutos de Mejoramiento y para la comunidad, se diseñaron estrategias que buscaban al descenso de la mortalidad infantil, el mejoramiento de las condiciones higiénicas de los hogares, de la vida doméstica, la alimentación, el vestido, el trabajo de la mujer, el de los niños y se lanzaron campañas de vacunación para evitar la propagación de enfermedades.

Durante los meses de febrero y marzo, los profesores misioneros tomaron cursos de entrenamiento en el edificio de la Escuela de Verano ubicada en la Universidad Nacional. En este programa, Moisés Sáenz impartía las materias de Filosofía de la educación y Crítica del trabajo efectuado por las Misiones en 1927. Especialmente para los profesores de educación física se asignaron las materias de baile regional, tenis y frontón, basquetbol, futbol, atletismo, beisbol, jockey, natación, juegos, organización deportiva, de festivales y escenografía. Estas actividades novedosas pero ajenas a la vida diaria de los pueblos fueron motivo de críticas que proclamaban una mayor atención a los deportes nacionales para evitar su desaparición con la implantación de deportes ajenos al medio y a la raza. Para contrarrestar la oposición a estas novedades, la SEP incluyó algunos libros en las bibliotecas ambulantes y se pidió a los profesores que trataran de despertar entre los misioneros, el esprit de corps, que a decir de su promotor, el profesor Sáenz, servía para mantener la energía y el entusiasmo en el trabajo así

\footnotetext{
${ }^{4}$ Adopto el termino de actividad física utilizado por Attali y Saint Martin quienes se centran en un sujeto que no se encuentra limitado únicamente por la actividad deportiva sino por las actividades propuestas en la escuela para atender el desarrollo físico del cuerpo (ATTALI; SAINT MARTIN, 2013, p. 54).
} 
como la unidad entre el grupo para despertar conciencia de la labor que desempeñaban entre la comunidad (SEP, 1928: 4) .

\section{LAS ACCIONES DE LA MISIÓN CULTURAL EN EL ESTADO DE MORELOS, MÉXICO}

Después de dos meses de entrenamiento, en 1928, las Misiones Culturales trabajaron en cinco zonas del País durante cuatro semanas. El municipio de Yautepec en el Estado de Morelos, ubicada a 60 kilómetros en el sur de la ciudad de México, fue seleccionado, bajo el argumento de que se trataba de una población desorganizada y con necesidades de rehabilitación porque estaba habitada por ejidatarios, con jornales insuficientes para dar trabajo a una población de casi 7 mil habitantes.

Se contaba con la experiencia porque dos años antes se había realizado una misión cultural en la misma zona, en el municipio de Cuautla. En este momento la comunidad conoció por primera vez las actividades físicas porque no había siquiera un espacio donde desarrollarlas. Bajo la dirección del inspector y del profesor de la materia, en una semana se barbechó y emparejó un terreno de la municipalidad. A pesar del clima caluroso y del esfuerzo que representó porque que aún había milpa, la comunidad colaboró para trazar los terrenos y en las canchas instalaron canastas rústicas de basquetbol. Las fotografías que acompañan los informes de las autoridades muestran el trabajo del maestro de cultura física pero también la colaboración de los vecinos.

Imagen 1.- Trabajos de barbecho y trazado en los terrenos deportivos del municipio de Cuautla.
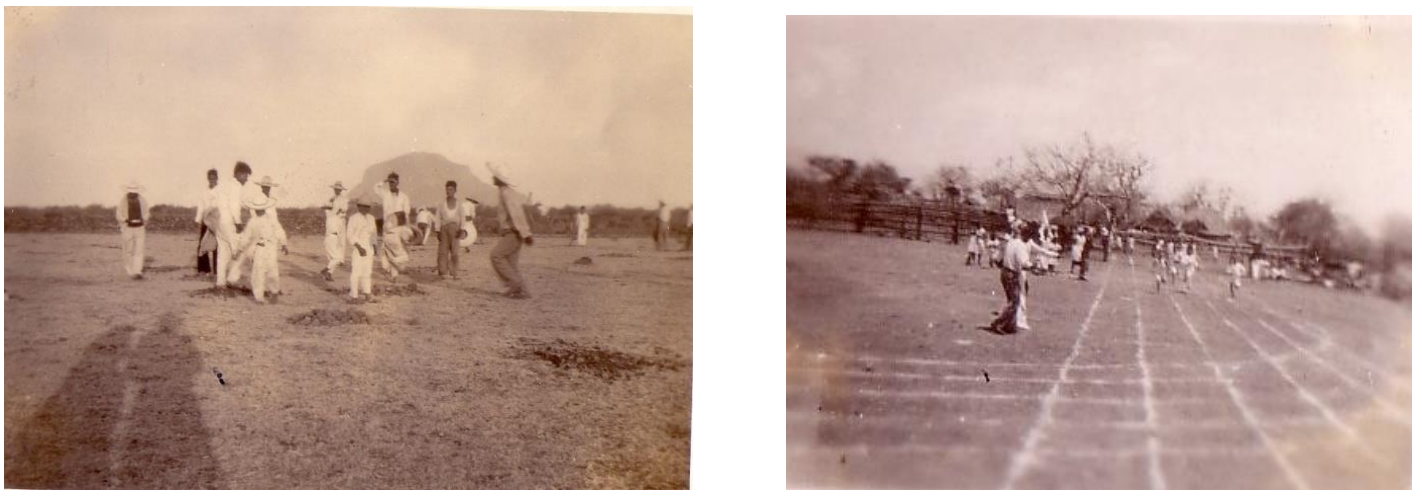

Fuente: AHSEP. Fondo Morelos. Caja 12.

\footnotetext{
${ }^{5}$ Circularon dos textos editados por la SEP, Juego de Pelota a mano "Tlaxtli" y Tribus de exploradores mexicanos de José escobar
} 
Las actividades se clausuraron con un encuentro atlético organizado por el maestro de educación física en el que se realizaron una serie de carreras de cien hasta ocho cientos metros, saltos de altura y de longitud. En general el programa muestra también una fuerte presencia de exhibiciones gimnásticas de parte de todos los grupos de la comunidad rural en el que se subraya el uso de un vestido especial para su ejercicio. Los carteles que fueron colocados en la población muestran también que las actividades estaban dirigidas a los jóvenes a quienes se invitaba a inscribirse hasta en tres eventos. En esta misión se privilegió la enseñanza del beisbol para los niños y del basquetbol para las niñas aún si, como lo señala Mary Kay Vaughan ${ }^{6}$, se reforzaron los roles de género pues eran las mujeres, las reinas, quienes colocaban las bandas de seda a los ganadores (AHSEP, 1926: exp.28; VAUGHAN, 2002, p. 45).

Estos nuevos saberes se enseñaron durante el tiempo en que los profesores misioneros permanecieron en la comunidad, cuando estaban en vías de partir la propia misión cultural se encargó de preservarlos con la construcción de un campo deportivo y de un pequeño parque infantil, donde se instalaron cuatro columpios y dos sube y baja para que habitantes siguieran realizando las mismas actividades novedosas. Ubicado en la plaza principal del pueblo, el parque fue inaugurado por las autoridades locales y desde entonces sirvió para disponer de los ratos de ocio de la comunidad rural.

Esta práctica se mantuvo en la propuesta de un nuevo proyecto educativo socialista. En 1935 se reorganizaron las zonas escolares en la entidad y el inspector, Arturo M. Arruti, quien había laborado antes como profesor, emprendió una serie de trabajos que muestran bien su interés por el desarrollo de zona que estaba a su cargo. Si bien el conflicto fanático religioso interfirió en las actividades diarias, el inspector Arruti estableció una serie de estrategias para cumplir con los propósitos. Una de estas fue organizar la Semana de Higiene en la que se realizaron exámenes antropométricos, mentales y profilácticos, se difundió una campaña para incentivar el uso del cepillo de dientes, la aplicación de vacunas y sobre todo la participaron de la comunidad en la vida deportiva.

Tal y como lo había diseñado Moisés Sáenz, quien había adaptado el proyecto de la YMCA para el desarrollo de las Misiones Culturales, diez años después seguían organizándose en las poblaciones rurales mexicanas, un conjunto de actividades deportivas y de entretenimiento (proyecciones de cine, conciertos, funciones de teatro, festivales) que respondían a un esquema en el que se privilegiaba la práctica del deporte como antídoto para

\footnotetext{
${ }^{6}$ Con sus propuestas Vaughan cumple su objetivo principal, a saber, "reconstruir una historia más rica, más sugerente, una historia no unidimensional, como parecían sugerir los informes, sino multivocal y multilinguística" (Vaughan, 2002: 42, 54)
} 
contrarrestar los efectos de la vida urbana pero también como vehículo para transmitir una serie de valores asociados a la integración de un país que aún mostraba tantas diferencias.

\section{UNA REFLEXIÓN FINAL}

Después del movimiento revolucionario de 1910, los profesores de las comunidades rurales contaban con muy pocas herramientas para desempeñar su labor; tenían una formación muy deficiente y pocos libros de consulta en la biblioteca. Aún así, los textos de Higiene y Medicina escrito por Luis G.León y La Salud del Niño, de Georges G.Croft fueron de gran ayuda en las labores de la escuela primaria del poblado de Mazatepec en el Estado de Morelos. Esta situación se revirtió en muy poco tiempo con la llegada de las Misiones Culturales a las comunidades rurales porque hacia la década de los treinta, los informes muestran que la gimnasia era un saber escolar que ocupaba un lugar importante dentro del currículo de la escuela primaria. En Mazatepec, un municipio que no había recibido directamente la influencia de las misiones culturales, había adoptado, en el transcurso de veinte años, la práctica de disciplinas como la lectura, la aritmética, el conocimiento de la naturaleza, la historia, la geografía, el dibujo, la costura, el canto y la gimnasia. En esta última materia los temas que se evaluaron fueron la formación en fila e hilera, los alineamientos, la numeración por pares y las carreras. Una actividad que confirma su valor en la formación del pequeño ciudadano pero también la participación de las niñas en estas actividades (AHM, 1932, s/d).

\section{BIBLIOGRAFÍA}

AHSEP Archivo Histórico de la Secretaría de Educación Pública. Fondo Morelos Colección Personal Sobresaliente Expedientes de Luis E. Ruiz (1878-1905) Expediente de Moisés Sáenz (1923-1926)

AHM Archivo Histórico de Mazatepec. Morelos

ATTALI, Michaël; Jean SAINT-MARTIN. Histoire du sport et histoire de l'education. En Terret Thierry et Tony Froissart (dir), Le sport, l'historien et l'histoire. Francia: Épure, 2013.

CHÁVEZ GONZÁLEZ, Mónica Lizbeth. La introducción de la educación física en México: representaciones sobre el género y el cuerpo, 1882-1928. Tesis de Maestría en Historia, El Colegio de San Luis, 2006, no publicada.

DAVILA, Paulí; NAYA, Luis Ma. (coords) Espacios y patrimonio histórico-educativo. España: Universidad del País Vasco, HMuseoa, EREIN, 2016 
MACÍAS CERVANTES, César Federico. Las prácticas deportivas (y las diversiones) en Guanajuato entre 1920 y 1960. Una propuesta de historia sociocultural. Tesis de Doctorado en Historia, ICSyH-BUAP, 2010, no publicada

LISBONA GUILLEN, Miguel. Mejorar la raza: cuerpo y deporte en el Chiapas de la Revolución Mexicana (1910-1940). Revista Relaciones, vol. XXVII, numero 105. Colegio de Michoacán, invierno 2006.

MA, Li. La place de la religion dans l'éducation par le YMCA des travailleurs chinois de la Grand Guerre. Guerres Mondiales et conflits contemporains, v. 3, n. 235, 2009. Disponible em: $\quad<$ https://www.cairn.info/revue-guerres-mondiales-et-conflits-contemporains-2009-3page-101.htm>. Consultado en septiembre 2015.

MARTÍNEZ MOCTEZUMA, Lucía. Las misiones culturales: un proyecto de educación y salud en el medio rural mexicano del siglo XX. RIDPHE_R, Revista Iberoamericana do Patrimônio Histórico-Educativo, v.2, n.3, p.101-116, 2016.

SÁENZ, Moisés. Lo esencial en la educación del estudiante. Revista Mexicana de Educación. Periódico mensual, número 6, Tomo 1, pp. 6-11, mayo 15 de 1920.

SEP. El sistema de escuelas rurales en México. México: Publicaciones de la Secretaría de Educación Pública, 1927.

SEP. Las Misiones Culturales en 1927. Las Escuelas Normales Rurales. México: Publicaciones de la Secretaría de Educación Pública, 1928.

SEP, Las Misiones Culturales 1932-1933. México: Publicaciones de la Secretaría de Educación Pública, 1933.

SPIVAK, Marcel. Quelques aperçus de la recherche en histoire de l'education physique et des sports en France. Histoire de l'Education, no.10, Ecole Normale Supérieure de Lyon, pp.119, abril1981.

VAUGHAN, Mary Kay. La historia de la educación y las regiones en México: cómo leer los informes de los inspectores escolares. En Galván Luz Elena, Civera y Escalante (coords) Debates y desafíos en la historia de la educación en México. México: El Colegio Mexiquense, A.C.-ISCEEM, 2002.

VÁZQUEZ BERNAL, Karina. Espacios, instituciones de educación indígena en México: la Casa del Estudiante Indígena y el Internado Indígena de Paracho (1926-1940). En Dávila Paulí y Anaya Luis María (coords) Espacios y Patrimonio histórico-educativo. España: Universidad del País Vasco-EREIN, p.135-148, 2016. 